\title{
HISTORIA Y REGIÓN: \\ LA HISTORIA REGIONAL DE CARA AL SIGLO XXI
}

\author{
History and Region: \\ Regional History on the twenty-first century
}

María Silvia Leoni*

\section{Resumen}

El artículo se inicia con algunas consideraciones conceptuales sobre región, regionalización y regionalismo y puntualiza algunos aspectos a considerar cuando estudiamos su construcción. Se realiza un repaso por el proceso de regionalización en la Argentina y más específicamente el caso del Nordeste. Este contexto permite explicar la situación actual de la historia regional a nivel latinoamericano y argentino, con las importantes innovaciones introducidas en las últimas décadas. Finalmente, una breve referencia a la relación de esta nueva historia con su enseñanza.

$$
<\text { Región }><\text { historia regional }><\text { regionalización }>
$$

\begin{abstract}
The article begins with some conceptual considerations on region, regionalization and regionalism and points out some issues to consider at the study of their construction. A review of the regionalization process in Argentina and more specifically the case of the Northeast is performed. This context helps to explain the current situation of the regional Latin American and Argentine history, with important innovations in recent decades. Finally, a brief reference to the relationship of this new history with teaching.

$$
<\text { Region }><\text { regional history }><\text { regionalization }>
$$

Recibido: 03/09/2015 // Aceptado: 06/11/2015
\end{abstract}

\footnotetext{
* Dra. en Historia. Facultad de Humanidades. UNNE. mariasilvia@gmx.net
} 
Para poder referirnos a la situación actual de la historia regional, partiremos de la ineludible pregunta ¿qué es una región? El concepto de región encierra una cuestión problemática, ya que no tiene una definición única y acabada. Remite en la actualidad a variadas definiciones y aproximaciones teóricas, propias de las distintas ciencias sociales y humanas: geografía, antropología, economía, entre otras.

Ante esta variedad de definiciones, la palabra región también aparece unida a otros términos: se habla de región geográfica, región económica, región histórica. Advertiremos que esta última -pese al uso del término histórica- no puede asimilarse a la noción de región que utiliza la historiografía hoy, ya que, como veremos, ha merecido críticas por parte de las perspectivas actuales.

Como punto de partida, consideramos que la región se construye sobre la dinámica de la relación hombre-espacio, es una construcción social, por lo cual debemos examinar los criterios utilizados para construirla.

La región entendida como producto de una "lógica social”, como "espacialidades diferenciales" -un concepto nacido a fines de la década de 1980, es considerada un sistema abierto, un objeto que se aborda mediante sucesivas aproximaciones que apuntan en su conjunto a la idea de totalidad (Girbal-Blacha y Cerdá, 2011). Por lo tanto, se han abandonado las concepciones esencialistas, que la consideraban dotada de una configuración persistente a través del tiempo, para referirla a un sistema o subsistema, a relaciones y efectos que determinan su conformación, continuidades y cambios. Se trata de un espacio heterogéneo, discontinuo y no necesariamente coincidente con los límites naturales o jurídicos.

Y aquí debemos plantearnos su vinculación con otras nociones, como las de frontera y espacio local. Unas pocas palabras sobre la noción de frontera, de gran utilidad en los estudios regionales. Entre los autores argentinos, Nidia Areces ha reflexionado sobre este tema e identifica la frontera como el espacio de intercambio, de flujo, de comunicación, por el que la comunidad se conecta con el exterior, en un sentido diametralmente opuesto al de la frontera como límite, correspondiente al paradigma del Estado nación. La frontera se configura por la expansión de una determinada sociedad sobre el espacio, de acuerdo con su propia dinámica interna. Esto lleva al establecimiento de variadas relaciones con las sociedades circundantes que a su vez, han construido sus propios espacios. La frontera es el área de contacto de formaciones sociales diversas (Areces, 2006). Pensemos en la importancia de estudiar el proceso de conformación de los espacios fronterizos en sus dimensiones política e institucional, que implica procesos de negociación entre regiones, al interior del Estado nacional, tanto como la imposición de reglas de juego desde el Estado nacional, cuando estos límites se fijan sin tomar en consideración la territorialidad. Las regiones existen dentro, entre y sobre los Estados constituidos y están siendo el principal factor de cambio estructural en las últimas décadas. Asimismo, al interior de una región existen unidades geográficas y sociales locales, y zonas fronterizas y transfronterizas internas, que pueden tener micro-dinámicas particulares (Hurtado, 2010). Por otro lado, el sentido de lo local (locus=perteneciente al lugar), tampoco tiene fronteras físicas ni administrativas inmutables. Es, como las otras, 
una categoría flexible. El espacio local ha adquirido importancia para la historiografía a partir del desarrollo de campos como el de la historia urbana.

En síntesis, el concepto de región se obtiene a partir de los resultados de la investigación y no antes. Se ha señalado que la región es una hipótesis por demostrar (Van Young). Es decir, la definición de región depende de la especificidad de la realidad histórica que se aborda; es entonces cuando se determina espacial y temporalmente.

\section{Región, regionalización y regionalismo}

Es importante atender a la distinción entre los conceptos de regionalización y de regionalismo. Regionalización se refiere a los esfuerzos estatales a través de una variedad de medidas para integrar la región dentro del estado y controlarla. Pensemos en los procesos de regionalización en Argentina desarrollados desde mediados del siglo XX. Regionalismo representa el esfuerzo por crear una conciencia y una ideología política dentro o en nombre de la región. Para Bourdieu (2006), el discurso regionalista es un discurso performativo, cuya finalidad es imponer como legitima una nueva definición de las fronteras y hacer conocer y reconocer la región así delimitada contra la definición dominante, que la ignora. Este acto de categorización ejerce poder por sí mismo: las categorías regionales instituyen una realidad usando el poder de revelación y de construcción ejercido por la objetivación en el discurso.

Los intelectuales han jugado un papel determinante en la tarea simbólica de construcción de la región, que, como señala Alejandro Benedetti, se combina, refuerza o contrapone, según las circunstancias, con otros factores que han intervenido en la producción de imaginarios regionales: la toponimia y la genealogía de categorías regionales, el discurso escolar, la literatura de viajeros, los discursos académico y político, la cartografía histórica y la iconografía oficial, la prensa y los medios de comunicación. Asistimos así a la construcción intelectual de un espacio al cual se le asignan características específicas.

El regionalismo se fundamenta en una exaltación de la patria chica; de la matria, como la denomina Luis Gonzalez y Gonzalez, y ha producido un tipo de historiografía, la historia local, que crea una mitología unificadora contraria a los proyectos unificadores nacionales (Betancourt, 2008).

\section{Región y estado nacional en la Argentina}

Desde una perspectiva política, podemos decir que la región es construida de manera similar a la nación, lo que lleva a discutir el nivel de invención frente al de fundación histórica. Como señala Einar Niemi (2000), estas unidades territoriales han sido construidas por los modernos procesos políticos de regionalización.

Las relaciones entre nación y región son centrales en nuestra historiografía a la hora de definir estos espacios. El vínculo con el espacio nacional y la convivencia entre regiones pueden generar relaciones de interdependencia tanto como de conflicto y 
también crean las disparidades regionales, en el sentido de que existen regiones centrales y regiones marginales o periféricas.

Distintas perspectivas historiográficas han buscado en la geografía claves para interpretar los conflictos no resueltos del proceso social argentino; claves como el despoblamiento y las distancias, el antagonismo litoral-interior o provincias-Nación. En la Argentina encontramos una potente matriz espacial en las explicaciones sobre la sociedad y la cultura nacionales; Adrian Gorelik (2001) observa que en esta matriz el mapa representa al cuerpo de la nación y se afirma que el hombre y la cultura son telúricos. Desde el siglo XIX, se advierte una larga tradición dentro del ensayismo que reconoce la clave de los males del país en el territorio y que equipara la palabra desierto con la ausencia de naturaleza, de pasado y de cultura.

Las diferencias regionales son tópicos frecuentes en los estudios sociales, pues existe consenso en torno a una visión sobre el territorio que pondera su diversidad en términos de oferta ambiental mientras observa negativamente sus disparidades internas en términos socio-económicos y poblacionales. Siguiendo nuevamente a Benedetti, diremos que, promediando el siglo XX, las políticas territoriales que buscaban solucionar los problemas de escaso crecimiento y desequilibrios sociales internos, apelaron a las teorías regionales que, a pesar de su exiguo éxito, dejaron en el mapa argentino algunas huellas.

Las regionalizaciones, señala Silvia Quintero (2002), podrían leerse en relación con dos horizontes problemáticos. Por un lado, con la necesidad de contar con instrumentos dotados de cierto consenso científico, para clasificar las riquezas y potencialidades de las distintas localizaciones del territorio nacional, con vistas a profundizar el proceso de inserción de la economía local en el espacio mundial. Por otro lado, como estrategias de integración y jerarquización de los sectores sociales que las regiones albergan o simbolizan.

Por su parte, los regionalismos en la Argentina no adquirieron el carácter de movimientos separatistas ni independentistas, ni cobraron gran protagonismo, siendo las identidades provinciales los más poderosos movilizadores de solidaridades que entraron en conflicto sistemáticamente con la territorialidad del estado nacional. Algunos provincialismos son muy marcados, como el caso correntino, lo cual incidiría negativamente en la construcción de una región como la nordestina. Chiaramonte (2008) señala como en el siglo XIX se había constituido una provincia-región en torno a la ciudad de Corrientes.

Por otro lado, en el pensamiento geográfico argentino se encuentran relatos regionales que arrancan en el pasado remoto, creando una ilusión de continuidad y permanencia, más allá de los procesos sociales de cambio; a ello colabora que en la toponimia regional hay nombres que tienen un origen prehispánico, como Chaco. Esta gran persistencia alimenta interpretaciones de larga duración, donde el surgimiento de las regiones muchas veces se rastrea, inclusive, hasta el neolítico (Benedetti, 2009).

La denominación Nordeste, en cambio, no es un término con raigambre histórica. El hoy llamado Nordeste argentino (provincias de Chaco, Corrientes, Formosa y 
Misiones) corresponde a un espacio de tardía incorporación al estado nacional -excepto el caso de la provincia de Corrientes-, pues gran parte de su superficie lo hizo en las últimas décadas del siglo XIX bajo el estatus de territorios nacionales, con todas las diferenciaciones y restricciones políticas que ello suponía para sus habitantes. Recién al promediar el siglo XX, Chaco, Formosa y Misiones adquirieron el rango de provincias argentinas.

El Nordeste no ha sido, por lo tanto, un espacio ni histórica ni geográficamente homogéneo; probablemente su característica común es su situación periférica con respecto al centro establecido en Buenos Aires. Más allá de ello, se advierten procesos diferenciados que tuvieron asiento en cada una de las provincias/regiones que hoy lo componen. Las particulares concepciones en torno a la hoy llamada región Nordeste condujeron a delinear sus propios límites y a buscar sus raíces históricas en diferentes momentos y circunstancias. De allí que se definieran distintas configuraciones de este espacio bajo denominaciones tales como región misionera, región chaqueña, Litoral argentino, Mesopotamia. El río Paraná ha jugado un papel en este contradictorio proceso de vinculación/separación entre los subespacios que conforman la región.

El dilema, que no pretendemos resolver en este momento, queda planteado, como se indica en el Prefacio de Fulgor del desierto verde (1985), del historiador chaqueño Guido Miranda, donde se pregunta "¿Gran Chaco o Nordeste argentino?"

\section{La historia regional}

El problema regional ha sido un tema recurrente en la historiografía latinoamericana. Una de las preocupaciones centrales ha sido cómo abordar el problema de la unidad nacional ante el fenómeno del regionalismo. El discurso integrador sobre la nación encubrió el problema regional. Paralelamente a los principios de la identidad nacional forjada por los liberales desde el siglo XIX, surgió una especie de "centralismo historiográfico" que negó lo heterogéneo y diverso, ocultó las diferencias espaciales existentes en el nivel local y regional, para crear y justificar la creación de las historias nacionales, como síntesis de las nacientes identidades nacionales latinoamericanas (Hurtado, 2010).

Las periodizaciones tradicionales se establecieron en los siglos XIX y XX siguiendo los modelos teóricos europeos, a partir de la dimensión del universo de análisis y la concepción dominante respecto al objeto de la historia. Ellas tomaron al marco nacional como el límite natural para el objeto de estudio del historiador, con un enfoque predominantemente político-institucional. Estas concepciones son las que se utilizaron para la periodización en la historiografía argentina; posteriormente, con la incorporación de los análisis económicos, se operó una nueva periodización, pero siempre siguiendo la tendencia a la homogeneización del espacio nacional.

Por su parte, en los estudios históricos regionales, tradicionalmente la región ha constituido un recorte espacial preexistente al inicio de la investigación. Han concebido a la región como región histórica, un constructo cultural resultante de un proceso 
histórico temprano que mantendría su identidad y homogeneidad desde la colonia hasta el presente. Vemos que no varían los supuestos que también establecían la legitimidad de la Historia Nacional. Al igual que ésta, la historia regional impuso un recorte territorial como previo al proceso histórico que en última instancia la instituyó a una como estado nación y a la otra como Región histórica (Mata, 2003).

Esta concepción tradicional de la historia presenta un doble problema. Por un lado, los trabajos sobre la problemática nacional, a veces 'generalizadores', se han sustentado en estudios empíricos de pocos casos y localizados en el área central. Por otra parte, muchos trabajos "regionales" tienen un grado de especificidad sobre lo local, sin establecer semejanzas y diferencias, que poco han contribuido a la historiografía argentina. En los medios académicos han sido frecuentes los cuestionamientos a la denominación de «historia regional» a trabajos que son historias provinciales, recorte espacial que atiende a limites políticos, más allá de que resulta útil para determinadas temáticas.

Por su parte, las nuevas concepciones sobre la región nos permiten revisar preconceptos existentes, propios de las historiografías nacionales, sobre el estado nacional, la sociedad nacional y el mercado nacional que se consideran plenamente conformados para fines del siglo XIX.

\section{La historia regional hoy}

Los procesos de globalización actuales tienen su contracara en los procesos de regionalización; se rescatan diferentes formas de ocupar el espacio y en tal sentido la región -con un enfoque antropológico social- se relaciona con las nuevas formas de organización del poder (Girbal-Blacha y Cerdá). La última globalización es un proceso de reorganización regional del planeta.

En el mundo de hoy, donde las identidades nacionales parecen diluirse, la historia identifica nuevos sujetos colectivos y construye nuevas categorías de análisis. En la transición al capitalismo global, la organización de las comunidades en el territorio se ha visto afectada por dos grandes procesos en apariencia divergentes. Uno es la regionalización, el fortalecimiento de las regiones en el ámbito nacional e internacional, afectando también a las regiones transfronterizas, como en el caso de las autonomías en España. El otro es la integración supranacional, que tiende a la construcción de grandes regiones, basadas a menudo en acuerdos económicos, pero con aspiraciones políticas, como la UE o el MERCOSUR.

Por otro lado, en una época de fragmentación del objeto de estudio de la historia o historia en migajas, según la conocida expresión de F. Dosse, se renueva el riesgo de identificar la historia regional con una historia localista. Por lo tanto, no se debe perder de vista "el contexto más amplio de cualquier región que pretende estudiar, a la par del empleo del método comparativo [para trascender] el análisis de regiones particulares mediante el estudio de interrelaciones entre diferentes áreas" (Hall cit. por Hurtado). La aplicación del método comparativo resulta fundamental para lograr este objetivo. 
En América Latina, de particular interés resultan los trabajos realizados por Alexander Betancourt que realiza estudios comparativos de regiones de México y Colombia.

La Historia Regional cuenta con antecedentes destacados en la experiencia de tendencias como la geohistoria, cuya obra paradigmática es El Mediterráneo de F. Braudel (1949), que propone una globalización regional.

En el caso latinoamericano, señala C. Aguirre Rojas (2003), a partir del exceso permanente de espacio que constituye una realidad de larga duración de la civilización latinoamericana, el hombre sólo ha podido afirmar su presencia orgánica y sistemática dentro de estos territorios de una manera desigual e irregular, dejando muchas regiones y espacios locales casi aislados o muy débilmente integrados a las dinámicas generales y luego nacionales de las más grandes concentraciones urbanas de población.

Esto explicaría el desarrollo de una potente historiografía regional latinoamericana, en las últimas tres décadas, que sorprende por la profundidad de sus enfoques y por la riqueza de sus resultados, lo mismo que por la variedad de los instrumentos y de los modos de aproximación analíticos y técnicos que ha sido capaz de desarrollar e inclusive de inventar. Este constituiría el aporte más importante de la historiografía de América Latina a los estudios históricos contemporáneos.

México encabezó el auge y la renovación historiográfica regional y local, seguido por Brasil, Venezuela, Argentina, Perú, Chile, Colombia, Bolivia, Costa Rica y Cuba.

La reflexión epistemológica y metodológica en el campo de los estudios históricos regionales se ha impuesto desde mediados de los noventa, lo que, según Aguirre Rojas, ha abierto grandes perspectivas para la investigación en el continente latinoamericano. Bajo la influencia de las corrientes historiográficas mundiales, los estudios regionales no se agotan en un modelo único, sino que hay una flexibilización de los modelos. Esta preocupación teórica se manifiesta en nuestro país en diversas compilaciones de trabajos que reflexionan sobre variadas aristas de este campo disciplinar.

Este desarrollo se ha visto favorecido también con el mejoramiento de las condiciones de los archivos y repositorios documentales o bibliográficos, las políticas estatales de descentralización de la educación superior, el intercambio académico entre los historiadores y científicos sociales en congresos y reuniones internacionales y los proyectos colectivos de investigación multidisciplinaria.

A la renovación de los estudios regionales han contribuido diferentes disciplinas sociales, entre las que encontramos la economía, la demografía y la sociología, primero y más recientemente a la antropología, la teoría literaria, los estudios culturales, las ciencias políticas. Se abandonaron los relatos políticos fácticos, considerados importantes para la construcción de la nación, para abocarse primero a los estudios económicos y sociales apoyados en diferentes teorías sociales y económicas, que obligaron a definir espacios inteligibles para el análisis, que excedían lo local y no se correspondían con los espacios delimitados por soberanías nacionales, jurisdicciones provinciales y regiones históricas. 
En nuestro país, en las últimas décadas, el énfasis con que tradicionalmente se homogeneizaba el discurso historiográfico desde Buenos Aires, comenzó a relativizarse al imponerse la historia regional, como una alternativa válida, considerada por muchos historiadores como una vía eficaz para superar la dicotomía historia nacional-historia provincial. En los estudios recientes de historia regional argentina, han dejado su huella las nuevas concepciones sobre el espacio y la región, que se apartan decididamente de la definición de una espacialidad en el orden natural. Heriberto Cairo señala que "un mapa no reproduce el mundo, lo construye y, más aún, naturaliza determinados hechos culturales" (Cairo, 2001).

Un primer ejemplo son los estudios de historia colonial andina de las últimas décadas que parten de la hipótesis de conformación regional y articulación en el espacio económico peruano formulada por Carlos Sempat Assadourian, que incluye en el análisis la circulación de mercancías, así como las relaciones políticas, económicas y socio-culturales.

Eric Van Young, autor de gran influencia en nuestra historiografía regional, propuso una visión de las regiones como determinadas por su relación con el mercado, con una perspectiva circulacionista, lo que le valió la crítica marxista por abandonar la esfera de la producción (Carbonari, 2009). Los estudios regionales desde una perspectiva económica han tenido un gran desarrollo; pensemos en los trabajos de Susana Bandieri sobre la Patagonia, los de Daniel Campi sobre las economías azucareras, los de Noemi Girbal sobre la región chaqueña.

Asimismo, se atiende a las construcciones de identidades colectivas en las cuales el discurso historiográfico juega un papel trascendental, en la medida en que, a través del rescate y del olvido del pasado, construye la memoria colectiva de una comunidad. Pero también tienen parte en ella la literatura, las costumbres, las formas de religiosidad popular, los rituales de las fiestas patrias, y muchas otras prácticas sociales. En El Gran Chaco (2006), Gastón Gordillo analiza la memoria social a la que define "como una práctica que contribuye a definir campos políticos, a internalizar y reformular discursos hegemónicos y a dar forma a la textura de configuraciones espaciales". Son entonces las experiencias las que definen la realidad a través de las configuraciones espaciotemporales. Los trabajos buscan la identificación de los sujetos y de las relaciones que establecen con y en el medio (económico, social, cultural, simbólico). Adquiere importancia el estudio de caso (familias, lazos parentales, lazos étnicos) para determinar estructuras de poder.

Desde la perspectiva de la nueva historia política, se ha pasado de lo factual a la preocupación por temas como el poder, la inestabilidad política, la representación, repensar el peronismo, los partidos políticos nacionales tradicionales, el sistema político, la prensa, la interpelación a los actores, el imaginario social y político, las prácticas, que son, entre otros, los temas-problemas presentes en muchos de los trabajos que se realizan en la actualidad, en los que cobran protagonismo los actores sociales, en tanto sujetos individuales y colectivos responsables de su acción (Leoni, 2013). 
En síntesis, ¿Qué le permite definir una región al historiador? No hay una definición apriorística del objeto de estudio. La región puede definirse a través de los espacios de circulación de bienes y personas, del desarrollo y dinámica de los mercados, de las articulaciones y relaciones de interdependencia, especializaciones, estructuras sociales y de poder, modalidades de acumulación, formación de clases y sistemas de dominación, con una mirada holística que supera la fragmentación entre historias sectoriales.

\section{Historia regional y educación}

Unas breves palabras sobre la relación entre HR y educación. Según el análisis realizado por Mariela Coudannes, la reforma educativa de la década del noventa en Argentina, que propuso articular la enseñanza de la historia nacional con la regional y latinoamericana, manejó un concepto de región en dos acepciones diferentes: una primera asociada a lo local y una segunda para ser aplicada al espacio definido por el Mercosur.

En la segunda acepción, justificó su lugar en el currículo planteando que la enseñanza debía servir para promover en las jóvenes actitudes y competencias favorables a la integración regional. Planteó desterrar los prejuicios nacionalistas que impiden reconocer los puntos de contacto entre las historias de los respectivos países, en pos de una identidad regional que no pierda de vista los particularismos culturales. También se planteó contextualizar y, de este modo, ampliar las posibilidades de comprensión de las problemáticas presentes en su realidad inmediata.

No obstante, a finales de la década sus impulsores reconocían la dificultad de la tarea de llevar a la enseñanza lo que se venía investigando, también con resultados incipientes; mayor aun en consecuencia era la dificultad de no contar con materiales didácticos acordes a las nuevas perspectivas.

Ya en el siglo XXI, en los diseños curriculares actuales, que buscan incluir las historias provinciales, no incorporan tampoco esta problematización.

Creemos, por ello, necesaria la formación de docentes a través de carreras como la Especialización en Historia Regional (UNNE), que permitan generar el espacio adecuado para que los cursantes puedan reflexionar y discutir no solo sobre el para qué y el cómo de la historia regional que se investiga, sino sobre la que se enseña y la que se debería enseñar a nuestros alumnos de los distintos niveles educativos.

\section{Referencias bibliograficas}

Aguirre Rojas, C. 2003. "El 'largo siglo XX' de la historiografía latinoamericana contemporánea: 1870-¿2025? Puntos de partida para su reconstrucción”. Historia Agenda, 1(3), http://www.cch.unam.mx/historiagenda/3/contenido/snh1.htm

Areces, N. 2006. "Pensar las fronteras". Mundo Agrario, vol. 7, n 13, segundo semestre de 2006. 
Benetti, A. 2009. "Los usos de la categoría región en el pensamiento geográfico argentino". En: Scripta Nova. Revista Electrónica de Geografía y Ciencias sociales, Barcelona, Universidad de Barcelona, vol. XIII, No 286, 15 de marzo s/p. http://www.ub.es/geocrit/sn/sn-286.htm

Betancourt Mendieta, A. 2008. Policromías de una región Procesos históricos y construcción del pasado local en el Eje Cafetero. México, Red de Universidades Públicas del Eje Cafetero - Alma Mater Coordinación de Ciencias Sociales y Humanidades de la Universidad Autónoma de San Luis Potosí.

Bourdieu, P. 2006. "La identidad y la representación: elementos para una reflexión crítica sobre la idea de región". En: Ecuador Debate, N 67, Centro Andino de Acción Popular, Quito, abril. http://www.dlh.lahora.com.ec/paginas/debate/ paginas/debate1637.htm

Bruniard, E. 1990. El Nordeste como región. Resistencia, Instituto de Investigaciones Geohistóricas- Conicet.

Cairo Carou, H. 2001. "Territorialidad y fronteras del estado-nación: Las condiciones de la política en un mundo fragmentado". En: Política y Sociedad, Madrid, $\mathrm{N}^{\circ}$ 36, pp. 29-38.

Carbonari, M. 2009. "De cómo explicar la región sin perderse en el intento. Repasando y repensando la Historia Regional". En: História Unisinos 13(1), enero/abril, pp. 19-34.

Chiaramonte, J. 2008. "Sobre el uso historiográfico del concepto de región". En: Estudios Sociales 35, segundo semestre

Coudannes, M. 2006. "Recorriendo el laberinto: historia regional santafesina y enseñanza". En: Anais Eletrônicos do VII Encontro Internacional da ANPHLAC, Campinas http://anphlac.fflch.usp.br/sites/anphlac.fflch.usp.br/files/mariela coudannes.pdf

Girbal- Blacha, N. y Cerdá, J. 2011. "Lecturas y relecturas sobre el territorio. Una interpretación histórica". En: Estudios Rurales. Publicación de Centro de Estudios de La Argentina Rural. Bernal, Vol. 1 No $^{\circ}$, 2011. ISNN: 2250. 55-78

Gordillo, G. 2006. En el Gran Chaco. Buenos Aires, Prometeo.

Gorelik, A. 2001. "Mapas de identidad. La imaginación territorial en el ensayo de interpretación nacional: de Ezequiel Martínez Estrada a Bernardo Canal Feijóo". En: Prismas, UNQ, No5.

Haesbaert, R. 2010. "Región, regionalización y regionalidad: cuestiones contemporáneas". En: Antares, $\mathrm{N}^{\circ} 3$, enero/junio.

Leoni, M. 2013. "Treinta años de historiografía política regional". En: Polhis, Boletín Bibliográfico Electrónico del Programa Buenos Aires de Historia Política, №12, $2^{\circ}$ semestre http://historiapolitica.com/datos/boletin/PolHis_12.pdf

Martina, E. 1968. “¿El Nordeste es una unidad regional?”. En: Boletín Filosófico. № 1, Resistencia, Facultad de Humanidades, Universidad Nacional del Nordeste, pp $52-55$. 
Mata, S. 2003. "Historia local, historia regional e historia nacional. ¿Una historia posible?". En: Revista Escuela de Historia, Universidad Nacional de Salta, año 2, Vol. $1, \mathrm{~N}^{\mathrm{o}} 2$.

Miranda, G. 1985. Fulgor del desierto verde. Resistencia, Región.

Niemi, E. 2000. "Region and regionalisation. Backround, trends, theories and models". En: $19^{\circ}$ International Congress of Historical Sciences. Oslo, http://www.oslo2000. uio.n./program/papers/s9/s9-niemi.pdf

Quintero, S. 2002. "Geografías regionales en la Argentina. Imagen y valorización del territorio durante la primera mitad del siglo XX". En: Scripta Nova Revista Electrónica de Geografía y Ciencias Sociales, Universidad De Barcelona, Vol. VI, No 127

Van Young, E. 1987. "Haciendo historia regional. Consideraciones metodológicas y teóricas". En: Anuario del IEHS, No 2, pp. 255-281.

Viales Hurtado, R. 2010. "La región como construcción social, espacial, política, histórica y subjetiva. Hacia un modelo conceptual/relacional de historia regional en América Latina". En: Geopolítica(s), vol. 1, №. 1, pp.157-172. 
